\title{
Chronic lymphocytic thyroiditis is associated with invasive characteristics of differentiated thyroid carcinoma in children and adolescents
}

\author{
Paschalia K Iliadou', Grigoris Effraimidis', Michalakis Konstantinos', \\ Panagiotou Grigorios', Periklis Mitsakis', Frideriki Patakiouta ${ }^{3}$ \\ and Kalliopi Pazaitou-Panayiotou ${ }^{1}$
}

${ }^{1}$ Department of Endocrinology, Theagenio Cancer Hospital, 2, Al Simeonidi Street, 54007 Thessaloniki, Greece, ${ }^{2}$ Endocrinology Section, Internal Medicine Department, Nykobing Falster Hospital, Nykobing Falster, Denmark and ${ }^{3}$ Department of Pathology, Theagenio Cancer Hospital, Thessaloniki, Greece
Correspondence should be addressed to K Pazaitou-Panayiotou Email

kpazaitou@in.gr

\begin{abstract}
Background: The association between chronic lymphocytic thyroiditis (CLT) and thyroid cancer is an interesting topic. The aim of the present study was to evaluate if demographic and histological characteristics as well as the long-term outcome of thyroid cancer was different in children and adolescents with and without CLT.

Methods: The medical records of children and adolescents ( $\leq 21$ years old) were reviewed. The following data were recorded: gender, year and age at diagnosis, family history of thyroid cancer, history of external radiation therapy, histological type (papillary and variants, follicular and variants), tumour size, multifocality, infiltration of thyroid parenchyma or surrounding soft tissues, vascular invasion, presence of lymph node and distant metastases. Information about the presence of $\mathrm{TgAb}$ and TPOAb was also collected.

Results: One hundred eight children and adolescents (median age 19.0, interquartile range 4.0 years) were diagnosed with differentiated thyroid carcinoma (DTC); 31 patients (28.7\%) presented histological characteristics compatible with CLT. Infiltration of thyroid parenchyma was more frequent in patients with CLT compared to patients without (74.2\% vs $48.1 \%$ respectively, $P=0.024)$. Familial papillary thyroid carcinoma (PTC) was more frequent in patients with CLT compared to those without CLT ( $20.7 \%$ vs $2.8 \%$ respectively, $P=0.009$ ). There was no better outcome with respect to the presence of CLT or not. Conclusions: Children and adolescents with CLT present more frequently familial PTC as well as thyroid cancer with invasive characteristics.
\end{abstract}

\section{Introduction}

Lymphocytic thyroiditis (LT) is a chronic inflammatory disease of the thyroid due to autoimmune responses. According to the International Classification of Diseases (ICD-10-CM Diagnosis Code E06.3), LT is defined as 'diffuse infiltration of the thyroid gland with lymphocytes, resulting in progressive destruction of the parenchyma and hypothyroidism.' Some physicians consider the presence of serum thyroid autoantibodies, e.g., thyroid peroxidase (TPOAb) and thyroglobulin antibodies (TgAb), as sufficient evidence for the diagnosis of chronic autoimmune thyroiditis. This is based on the observation that thyroid antibodies correlate well with the presence of lymphocytic infiltrates found during histological examination of the thyroid in individuals without prior history of thyroid failure.

During the last three decades, an increase in the annual frequency of chronic LT (CLT) was observed. In a retrospective study done in the University Hospital of Messina, Italy, from January 1975 to December 2005, it was found that CLT has become more frequent after 1994

Published by Bioscientifica Ltd. 
and a marked increase in the annual frequency was observed thereafter reaching 64\% between 1996 and 1997 and 42\% between 1997 and 1998. Subsequently, the increase continued but with a lower rate and reached a minimal increase rate of 3\% between 2004 and 2005. In the same work it was found that affected patients were relatively younger compared to the past (1). An increase in the annual frequency of CLT, but to a lesser extent, was also observed in Catania, which is located south of the Messina province, between 1995 and 2005 (2). Different environmental modified factors could probably explain this marked increase that occurred in a relatively short period of time (1).

Regarding the association of CLT with other thyroid diseases, it has been observed that the most common condition is papillary thyroid carcinoma (PTC) (3), and since the first report in 1955 by Dailey et al. (4), studies regarding this association are ongoing. Coexistence of the two diseases ranges between 0.5 and $58 \%(2,3,5,6)$. This wide distribution of coexisting CLT and PTC may at least in part be due to differences in histological examination and criteria of autoimmunity characterization, patient selection or indications for thyroidectomy, population background and, mainly, environmental factors (2). A recent meta-analysis demonstrated that the occurrence rate of CLT was 2.8 times higher in patients with PTC compared to patients with benign thyroid diseases (7) and 2.4 times higher in patients with PTC than in those with other histopathological types (7). Albeit indirectly, these results suggest that patients with CLT might well present a stronger predisposition toward the development of PTC. Furthermore, CLT and PTC share some common epidemiological features, such as the relationship to ionizing radiation exposure and dietary iodine, as well as some molecular features (6).

Regarding the influence of CLT on the evolution of PTC, different studies showed a protective effect of thyroid autoimmunity, i.e., less aggressive disease at presentation and/or better long-term outcome $(8,9,10,11)$. However, multivariate analysis in other studies revealed that CLT was not an independent predictor of a better outcome of differentiated thyroid carcinoma (DTC) $(12,13,14)$. The above-mentioned findings refer to patients regardless of age, and there are no published studies focusing on the characteristics and outcome of thyroid cancer in children with and without CLT. However, the frequency of PTC in children and adolescents with CLT has been evaluated in two published studies. In the first one, 11 of 365 children and adolescents (3\%) with CLT were diagnosed with PTC (15), and in the second study, three of 228 Greek children and adolescents (1.3\%) with CLT presented PTC (16).

The aim of the present study was to evaluate if demographic and histological characteristics as well as the long-term outcome of thyroid cancer were different in children and adolescents with and without autoimmune thyroiditis, taking into consideration that information in the literature is scarce.

\section{Subjects and methods}

The medical records of children and adolescents with histologically proven DTC followed in our hospital were reviewed. The following data were recorded: presenting signs or symptoms of the disease, year of diagnosis of thyroid cancer, age at diagnosis, gender, family history of thyroid cancer, history of external radiation therapy to the head and neck, type of surgery, histological type (papillary and variants, follicular and variants), tumour size, number of tumour foci (unifocal/multifocal), thyroid capsule invasion, vascular invasion, infiltration of thyroid parenchyma or surrounding soft tissues and presence of lymph node metastases as well distant metastases. Information about the presence of $\mathrm{TgAb}$ and TPOAb was also collected. Postoperative staging was done based on the tumour, nodes, metastases (TNM) system as proposed by the American Joint Committee on Cancer (AJCC). Histological criteria used for the diagnosis of LT were diffuse lymphocytic infiltration with the presence of germinal centres in the stroma, presence of thyroid follicles with oxyphilic change of the follicular epithelium and presence of fibrosis.

Patients were divided in two groups based on the presence (group A) or not (group B) of LT. Data analysis was performed using the statistical package SPSS (version 17.0; SPSS, Inc.). The Kolmogorov-Smirnov or ShapiroWilk test was used to assess the normality of distribution of continuous variables. Continuous variables not normally distributed are described by median and interquartile range (IQR). An independent samples $T$-test or Mann-Whitney $U$-test was used to test for between-groups differences in continuous variables. Data for categorical variables are presented as absolute numbers and percentages. The $\chi^{2}$-test was used to assess between-groups differences in categorical variables. In all cases a $P$ value $<0.05$ was considered significant. All data were analysed anonymously. The study was approved by the Institutional Review Board. 


\section{Results}

One hundred eight children and adolescents with DTC (82 females, 76\%) were identified. Patients first visited their pediatrician or general practicing doctors because of a family history of thyroid diseases or due to a cervical mass accidentally found by themselves or their parents or by a physician in a routine examination. All had a short follow-up of 4-10 months before thyroidectomy, which was performed as they were found to harbour a nodule suspicious (fine needle aspiration (FNA) and/or ultrasound) for malignancy. No patient had received external radiation therapy in the neck or head for the treatment of another primary malignancy. Preoperatively, TgAb and TPOAb were measured in 98 patients and found to be positive in 27 (27.6\%). Near total or total thyroidectomy was performed in all patients with central and/or lateral lymph node dissection in 69 of them.

Histology revealed papillary carcinoma in the majority of the cases (93.5\%) (Table 1). Median (IQR) tumour size was 15.5 (21.0) $\mathrm{mm}$ (range 5-70 mm). Although not statistically significant, tumour size tended to be larger in males in comparison to females (20.0 (30.0) vs 13.5 (17.0) $\mathrm{mm}, P=0.063)$. There were no differences between males and females regarding age at diagnosis, familial and sporadic cancer, histological type or other histological characteristics. Classification according to the TNM system showed T1: 58, T2: 19, T3: 27 and T4: 4 patients; N0: 50, N1a: 17, N1b: 41 patients; and M0: 98, M1: 10 patients.

Of the 108 patients, 31 (28.7\%) presented histological characteristics compatible with CLT and these patients comprised group A. Among them, thyroid autoantibodies were found to be positive in 25 (80.6\%); 77 patients without histological characteristics compatible with CLT comprised group B. Among patients of group B, two were found with positive TgAb but none had TPOAb (Fig. 1). The percentage of CLT was similar in males and females (19.2 and $31.7 \%$ respectively, $P=0.220$ ) and frequency was also similar in patients $>18$ years old compared to those $\leq 18$ years old (31.3 and $25.0 \%$ respectively, $P=0.481$ ). Thyroid carcinoma presented intrathyroidal invasion more frequently in group A compared to group B (74.2\% vs $48.1 \%$ respectively, $P=0.024)$. Other clinical and histological characteristics did not differ between groups A and $B$ (Table 1).

Thirty-nine patients were found to have tumours $\leq 10 \mathrm{~mm}$, ten of them with CLT. No differences were found regarding the percentage of CLT in patients with tumour size $\leq 10 \mathrm{~mm}$ compared to those with size $>10 \mathrm{~mm}$. Familial PTC was found more frequent in group A compared to group B (20.7 vs 2.8\% respectively, $P=0.009$ ). Median duration of follow-up was 54 months (range 7-324) for group A and 53 months (range 8-347) for group B $(P=0.732)$. From the total cohort, 48 patients were followed for 5 years and 27 of those for at least 10 years; 38 (79.2\%) of the former and $22(81.5 \%)$ of the latter were found free of the disease. No differences were observed regarded the disease status in patients with or without CLT after 5 or 10 years of follow-up $(P=0.948$ and $P=0.738$ respectively).

\section{Discussion}

In the present study we evaluate clinical and histological characteristics in 108 children and adolescents with DTC according to the presence or not of CLT. Out of the overall cohort, 31 patients (28.7\%) presented histological characteristics compatible with CLT. We found that the infiltration of the thyroidal parenchyma and familial PTC was more frequent in patients with CLT compared to those without. However, no better outcome was observed in regards to the absence or presence of CLT. To our knowledge, this is the largest (regarding the number of patients) study in terms of examining thoroughly the relationship between CLT and DTC in children and adolescents.

A significant increase in the number of Hashimoto's thyroiditis associated with PTC was observed in surgical specimens during the last two decades as reported in a recently published study written to celebrate a century since the identification of Hashimoto's thyroiditis. This association remains unexplained as it is difficult to answer if it represents a simple co-occurrence of two relatively common thyroid conditions or if it indicates a true causeeffect relationship (3). There have been many studies conducted to elucidate this association. One of these supported that the oncogenic RET/PTC rearrangements, a specific genetic alteration in PTC patients, are present in Hashimoto's thyroiditis (17). RET/PTC mutations are reported in about $68-95 \%$ of Hashimotos' patients $(18,19,20)$. Moreover, it is reported that the RET/PTC1 oncogene, when exogenously expressed in primary normal human thyrocytes, induces the expression of a large set of genes involved in inflammation and tumour invasion (21). Other studies suggest a role for inflammation in the development of thyroid cancer. In one of these, it is reported that patients with Hashimoto's thyroiditis were three times more likely to have thyroid cancer, and it suggested a possible molecular mechanism for thyroid carcinogenesis through the increased 
Table 1 Clinical characteristics of the patients/participants and histological characteristics of their tumours. Group A: patients with histological characteristics compatible with CLT. Group B: patients without histological characteristics compatible with CLT. Data are presented as median (interquartile range in parentheses) or absolute numbers (percentages in parentheses).

\begin{tabular}{l} 
Characteristics \\
Age at diagnosis (years) \\
Gender \\
Male \\
Female \\
Familial \\
Histologic type \\
Papillary classic type \\
Papillary-follicular variant \\
Papillary-other variants \\
Follicular \\
Tumour size (mm) \\
Bilateral \\
Multifocal \\
Nodule capsule invasion \\
Thyroid parenchyma infiltration \\
Thyroid capsule invasion \\
Infiltration of surrounding soft tissues \\
Lymph node metastases \\
Vascular invasion \\
Lymph vessel invasion \\
Vascular embolus \\
Metastasis \\
Number of patients with measured autoantibodies \\
Number of patients with positive antibodies \\
Treatment modalities \\
Surgery \\
TT \\
TT and LND \\
RAl \\
Number of treatments \\
Total dose (mCi) \\
Duration of follow-up (months) \\
Patient's condition at last follow-up \\
Free of disease \\
Death \\
Distant metastases \\
Local disease \\
Follow-up $<10$ months \\
No follow-up \\
\hline
\end{tabular}

\begin{tabular}{c}
\hline Group A $(n=31)$ \\
\hline $20.0(4.0)$ \\
$5(16.1)$ \\
$26(83.9)$ \\
$6(19.4)$ \\
$19(61.3)$ \\
$8(25.8)$ \\
$2(6.5)$ \\
$2(6.5)$ \\
$13.0(22.0)$ \\
$8(25.8)$ \\
$13(41.9)$ \\
$5(100.0)$ \\
$23(74.2)$ \\
$12(38.7)$ \\
$7(22.6)$ \\
$17(85.0)$ \\
$7(22.6)$ \\
$3(9.7)$ \\
$3(10.0)$ \\
$2(6.5)$ \\
$29(93.5)$ \\
$25(86.2)$ \\
\end{tabular}

\begin{tabular}{ccc}
\hline Group B $(n=77)$ & & P value \\
\hline $19.0(5.0)$ & & 0.217 \\
$21(27.3)$ & 0.220 \\
$56(72.7)$ & & 0.009 \\
$2(2.6)$ & & 0.953 \\
& &
\end{tabular}

TT, total (or near total) thyroidectomy; LND, lymph node dissection; RAI, radioactive iodine.

aVariants: trabecular $(n=3)$, sclerosing $(n=3)$, solid $(n=1)$.

${ }^{\mathrm{b}}$ Among the 23 patients with nodule capsule.

'Among the 69 patients who underwent lymph node dissection.

${ }^{\mathrm{d}}$ Among patients with measured autoantibodies.

e In both groups patients received 1311, one to five times (maximum dose of $600 \mathrm{mCi}$ ).

expression of the phosphatidylinositol 3-kinase (PI3K)/Akt pathway in patients with Hashimoto's thyroiditis (22), while another demonstrated that stepwise increments in overexpression of both COX-2 and iNOS were demonstrated in epithelial cells in HT, follicular adenoma and papillary thyroid cancer while normal thyroid tissue showed little expression (23).

The frequency of LT in children with DTC was reported in a few published studies, ranging from 6.3 to $43.0 \%$
$(24,25,26)$. Nevertheless, these studies do not clarify which histopathological criteria were used by the authors to determine the presence of CLT. In particular, in an older study of 48 children and adolescents with thyroid cancer, only $3(6.3 \%)$ had CLT (24). In a more recent study, 23 of 54 (43\%) children with DTC (48 PTC and six FTC) had 'background thyroiditis' on histological examination (25), and in another one, 16 of 90 (17.8\%) children and adolescents with DTC (78 PTC and 12 FTC) showed 'diffuse 


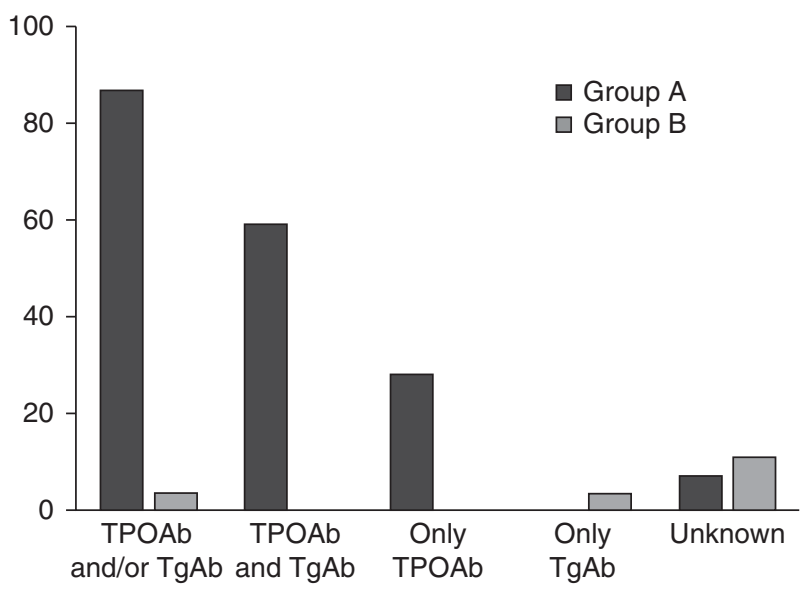

\section{Figure 1}

Proportion of patients with TPOAb and/or TgAb, TPOAb and $\operatorname{TgAb}$, only TPOAb and only TgAb in serum in the group of patients with measured thyroid antibodies. Proportion of unknown thyroid antibodies status in the entire study population. Group A: patients with histological characteristics compatible with CLT. Group B: patients without histological characteristics compatible with CLT.

thyroiditis' on histological examination (26). In the present study we found that $28.6 \%$ of the patients presented with CLT. Differences in patient selection might have contributed to this variability that was associated with geographic and ethnic diversity in the prevalence of CLT and DTC.

The prognostic value of thyroid autoimmunity for the outcome of DTC has also been studied, but with conflicting results. A number of studies showed a protective effect of thyroid autoimmunity, i.e., less aggressive disease at presentation and/or better long-term outcome $(8,9,10,11)$. However, multivariate analysis in other studies revealed that CLT was not an independent predictor of better outcome of DTC $(12,13,14)$. Nevertheless, the above-mentioned studies report data on adults only or both adults and children, and in the latter case patients are not divided in two groups based on their age; therefore, it is impossible to extract information regarding children. To our knowledge, no studies have been carried out in children and adolescent populations regarding the prognostic value of thyroid autoimmunity for the outcome of DTC. On the other hand, some studies report the prevalence of DTC in children with CLT, which was found to be $1.3-3.0 \%(15,16)$. In one of those studies (15), more girls were affected by CLT, but males with CLT had an increased risk to develop thyroid cancer (odds ratio (OR), 2.95; 95\% confidence interval (CI), 1.44-6.20).
It has been reported that lymphocytes migrate around a malignant tumour in an effort to restrict disease extension (27). This process, known as peritumoural infiltration, is different from that described in CLT as diffuse lymphocytic infiltration. In a small study with nine patients aged $<20$ years with PTC (all with serum negative antibodies), Kamma et al. (28) found that lymphocytic infiltration was observed in more aggressive cases, predominantly near the tumour, and the degree of infiltration was correlated with the extension of carcinoma. They concluded that lymphocytic infiltration around the tumour is an immunologic reaction induced by antigens of the carcinoma itself. Gupta et al. (29) demonstrated that among 48 children and adolescents with thyroid carcinoma (39 PTC and nine FTC), those who had a high number of proliferating lymphocytes, demonstrated by the proliferating cell nuclear antigen Ki-67, had a better disease-free survival than those with lower proliferating numbers. In the present study, cases with peritumoural lymphocytic infiltration were scarce and therefore not discussed as a third group.

In our study we found that familial PTC is more frequent in children with CLT in comparison to those without CLT. A possible explanation is that children with a positive family history of CLT are more frequently examined for thyroid disease (or more frequently referred to specialists).

It is difficult to answer if children with CLT should be closely monitored for the development of thyroid cancer, as some studies suggested that the prevalence of PTC may be higher in children with CLT (16). As children with CLT are followed up due to functional thyroid disorders (30), the additional examination by ultrasonography could detect nodule(s) with suspicious characteristics.

A limitation of the present study is its retrospective nature. Our study is the largest one focusing on different histological characteristics and evolution of DTC in patients with and without CLT, although it seems to include a relatively small number of patients. Considering that thyroid cancer is relatively rare in children and that both paediatric and adult endocrinologists follow these patients, it is evident that a single centre may not have a large number of patients for treatment and follow-up. Multi-centre studies with a larger number of patients and long follow-up duration are needed to evaluate disease course.

In conclusion, in this retrospective study it was shown that children and adolescents with CLT present more frequently invasive DTC and familial PTC. No better outcome of the cancer was observed between children 
with CLT in comparison to those without. Multicentre, epidemiological studies are needed to confirm our results. Paediatricians or primary care physicians should be aware of this association when diagnosing or treating children with CLT.

\section{Declaration of interest}

The authors declare that there is no conflict of interest that could be perceived as prejudicing the impartiality of the research reported.

\section{Funding}

This research did not receive any specific grant from any funding agency in the public, commercial or not-for-profit sector.

\section{Acknowledgements}

We are very grateful to Prof. W M Wiersinga for his critical reading of the manuscript, helpful and constructive comments and fruitful and insightful discussions.

\section{References}

1 Benvenga S \& Trimarchi F. Changed presentation of Hashimoto's thyroiditis in North-Eastern Sicily and Calabria (Southern Italy) based on a 31-year experience. Thyroid 200818 429-441. (doi:10.1089/thy. 2007.0234)

2 Latina A, Gullo D, Trimarchi F \& Benvenga S. Hashimoto's thyroiditis: similar and dissimilar characteristics in neighboring areas. Possible implications for the epidemiology of thyroid cancer. PLOS ONE 20138 e55450. (doi:10.1371/journal.pone.0055450)

3 Caturegli P, De Remigis A, Chuang K, Dembele M, Iwama A \& Iwama S. Hashimoto's thyroiditis: celebrating the centennial through the lens of the Johns Hopkins hospital surgical pathology records. Thyroid 201323 142-150. (doi:10.1089/thy.2012.0554)

4 Dailey ME, Lindsay S \& Skahen R. Relation of thyroid neoplasms to Hashimoto disease of the thyroid gland. A.M.A. Archives of Surgery 1955 70 291-297. (doi:10.1001/archsurg.1955.01270080137023)

5 Feldt-Rasmussen U \& Rasmussen AK. Autoimmunity in differentiated thyroid cancer: significance and related clinical problems. Hormones 20109 109-117. (doi:10.14310/horm.2002.1261)

6 Cunha LL, Ferreira RC, Marcello MA, Vassallo J \& Ward LS. Clinical and pathological implications of concurrent autoimmune thyroid disorders and papillary thyroid cancer. Journal of Thyroid Research 20112011 387062. (doi:10.4061/2011/387062)

7 Lee JH, Kim Y, Choi JW \& Kim YS. The association between papillary thyroid carcinoma and histologically proven Hashimoto's thyroiditis: a meta-analysis. European Journal of Endocrinology 2013168 343-349. (doi:10.1530/EJE-12-0903)

8 Dvorkin S, Robenshtok E, Hirsch D, Strenov Y, Shimon I \& Benbassat CA. Differentiated thyroid cancer is associated with less aggressive disease and better outcome in patients with coexisting Hashimotos thyroiditis. Journal of Clinical Endocrinology and Metabolism 201398 2409-2414. (doi:10.1210/jc.2013-1309)

9 Loh KC, Greenspan FS, Dong F, Miller TR \& Yeo PP. Influence of lymphocytic thyroiditis on the prognostic outcome of patients with papillary thyroid carcinoma. Journal of Clinical Endocrinology and Metabolism 199984 458-463. (doi:10.1210/jcem.84.2.5443)
10 Kashima K, Yokoyama S, Noguchi S, Murakami N, Yamashita H, Watanabe S, Uchino S, Toda M, Sasaki A, Daa T et al. Chronic thyroiditis as a favorable prognostic factor in papillary thyroid carcinoma. Thyroid 19988 197-202. (doi:10.1089/thy.1998.8.197)

11 Singh B, Shaha AR, Trivedi H, Carew JF, Poluri A \& Shah JP. Coexistent Hashimoto's thyroiditis with papillary thyroid carcinoma: impact on presentation, management, and outcome. Surgery 1999126 1070-1076; discussion 1076-1077. (doi:10.1067/msy.2099.101431)

12 Kebebew E, Treseler PA, Ituarte PH \& Clark OH. Coexisting chronic lymphocytic thyroiditis and papillary thyroid cancer revisited. World Journal of Surgery 200125 632-637. (doi:10.1007/ s002680020165)

13 Kim EY, Kim WG, Kim WB, Kim TY, Kim JM, Ryu JS, Hong SJ, Gong G \& Shong YK. Coexistence of chronic lymphocytic thyroiditis is associated with lower recurrence rates in patients with papillary thyroid carcinoma. Clinical Endocrinology 200971 581-586. (doi:10.1111/ j.1365-2265.2009.03537.x)

14 Jeong JS, Kim HK, Lee CR, Park S, Park JH, Kang SW, Jeong JJ, Nam KH Chung WY \& Park CS. Coexistence of chronic lymphocytic thyroiditis with papillary thyroid carcinoma: clinical manifestation and prognostic outcome. Journal of Korean Medical Science 201227 883-889. (doi:10.3346/jkms.2012.27.8.883)

15 Corrias A, Cassio A, Weber G, Mussa A, Wasniewska M, Rapa A, Gastaldi R, Einaudi S, Baronio F, Vigone MC et al. Thyroid nodules and cancer in children and adolescents affected by autoimmune thyroiditis. Archives of Pediatrics \& Adolescent Medicine 2008162 526-531. (doi:10.1001/archpedi.162.6.526)

16 Skarpa V, Kousta E, Tertipi A, Anyfandakis K, Vakaki M, Dolianiti M, Fotinou A \& Papathanasiou A. Epidemiological characteristics of children with autoimmune thyroid disease. Hormones $2011 \mathbf{1 0}$ 207-214. (doi:10.14310/horm.2002.1310)

17 Arif S, Blanes A \& Diaz-Cano SJ. Hashimoto's thyroiditis shares features with early papillary thyroid carcinoma. Histopathology 200241 357-362. (doi:10.1046/j.1365-2559.2002.01467.x)

18 Wirtschafter A, Schmidt R, Rosen D, Kundu N, Santoro M, Fusco A, Multhaupt H, Atkins JP, Rosen MR, Keane WM et al. Expression of the RET/PTC fusion gene as a marker for papillary carcinoma in Hashimoto's thyroiditis. Laryngoscope 1997 107 95-100. (doi:10.1097/ 00005537-199701000-00019)

19 Sheils OM, O'Eary JJ, Uhlmann V, Lattich K \& Sweeney EC. ret/PTC-1 activation in Hashimoto thyroiditis. International Journal of Surgical Pathology 20008 185-189. (doi:10.1177/ 106689690000800305)

20 Rhoden KJ, Unger K, Salvatore G, Yilmaz Y, Vovk V, Chiappetta G, Qumsiyeh MB, Rothstein JL, Fusco A, Santoro M et al. RET/papillary thyroid cancer rearrangement in nonneoplastic thyrocytes: follicular cells of Hashimoto's thyroiditis share low-level recombination events with a subset of papillary carcinoma. Journal of Clinical Endocrinology and Metabolism 200691 2414-2423. (doi:10.1210/jc.2006-0240)

21 Borrello MG, Alberti L, Fischer A, Degl'innocenti D, Ferrario C, Gariboldi M, Marchesi F, Allavena P, Greco A, Collini P et al. Induction of a proinflammatory program in normal human thyrocytes by the RET/PTC1 oncogene. PNAS 2005102 14825-14830. (doi:10.1073/pnas. 0503039102)

22 Larson SD, Jackson LN, Riall TS, Uchida T, Thomas RP, Qiu S \& Evers BM. Increased incidence of well-differentiated thyroid cancer associated with Hashimoto thyroiditis and the role of the PI3k/Akt pathway. Journal of the American College of Surgeons 2007 204 764-773; discussion 773-765. (doi:10.1016/j.jamcollsurg.2006. 12.037)

23 Nose F, Ichikawa T, Fujiwara M \& Okayasu I. Up-regulation of cyclooxygenase-2 expression in lymphocytic thyroiditis and thyroid tumors: significant correlation with inducible nitric oxide synthase. American Journal of Clinical Pathology 2002117 546-551. (doi:10.1309/ 9CCJ-XQ8P-PMFM-M65K) 
24 Danese D, Gardini A, Farsetti A, Sciacchitano S, Andreoli M \& Pontecorvi A. Thyroid carcinoma in children and adolescents. European Journal of Pediatrics 1997156 190-194. (doi:10.1007/ s004310050580)

25 O'Gorman CS, Hamilton J, Rachmiel M, Gupta A, Ngan BY \& Daneman D. Thyroid cancer in childhood: a retrospective review of childhood course. Thyroid 201020 375-380. (doi:10.1089/thy. 2009.0386)

26 Park S, Jeong JS, Ryu HR, Lee CR, Park JH, Kang SW, Jeong JJ, Nam KH, Chung WY \& Park CS. Differentiated thyroid carcinoma of children and adolescents: 27-year experience in the Yonsei University Health System. Journal of Korean Medical Science 201328 693-699. (doi:10.3346/jkms.2013.28.5.693)

27 Fridman WH, Galon J, Pages F, Tartour E, Sautes-Fridman C \& Kroemer G. Prognostic and predictive impact of intra- and peritumoral immune infiltrates. Cancer Research 201171 5601-5605. (doi:10.1158/ 0008-5472.CAN-11-1316)

28 Kamma H, Fujii K \& Ogata T. Lymphocytic infiltration in juvenile thyroid carcinoma. Cancer 198862 1988-1993. (doi:10.1002/ 1097-0142(19881101)62:9<1988::AID-CNCR2820620919>3.0. CO;2-0)

29 Gupta S, Patel A, Folstad A, Fenton C, Dinauer CA, Tuttle RM, Conran R \& Francis GL. Infiltration of differentiated thyroid carcinoma by proliferating lymphocytes is associated with improved disease-free survival for children and young adults. Journal of Clinical Endocrinology and Metabolism 200186 1346-1354.

30 Gopalakrishnan S, Chugh PK, Chhillar M, Ambardar VK, Sahoo M \& Sankar R. Goitrous autoimmune thyroiditis in a pediatric population: a longitudinal study. Pediatrics 2008122 e670-674. (doi:10.1542/peds. 2008-0493)

Received 27 November 2014

Revised version received 15 June 2015

Accepted 8 September 2015 